\title{
IN-SERVICE DESIGN \& PERFORMANCE PREDICTION OF ADVANCED FUSION MATERIAL SYSTEMS BY COMPUTATIONAL SIMULATION
}

\author{
Final Project Report \\ (for the DOE Grant: DE-FG03-01ER54626) \\ Technology Division \\ Office of Fusion Energy Sciences \\ Office of Energy Research \\ Department of Energy \\ Germantown, MD 20874-1290
}

For the Period

March 15, 2001 - March 14, 2004

Nasr M. Ghoniem, Principal Investigator

Mechanical and Aerospace Engineering Department, UCLA 


\section{Contents}

1 Introduction and Background $\quad 3$

2 Radiation Hardening and Plastic Instabilities 3

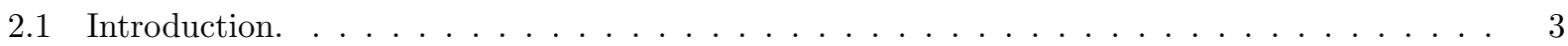

2.2 Dislocation Unlocking from Cluster Atmospheres . . . . . . . . . . . . . . . . . . 4

2.3 Dislocation Interaction with Sessile Defect Clusters . . . . . . . . . . . . . . . . . 4

2.4 Interaction with Stacking Fault Tetrahedra (SFTs) in FCC Metals . . . . . . . . . . . . 5

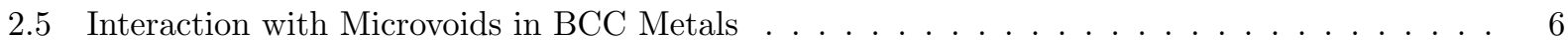

2.6 Conclusions and Future Outlook $\ldots \ldots \ldots \ldots \ldots \ldots \ldots$

3 Non-equilibrium Phase Transitions $\quad 9$

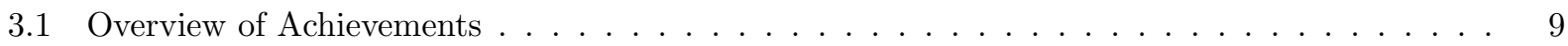

3.2 Publications on Phase Evolution $\ldots \ldots \ldots \ldots \ldots \ldots \ldots$

4 Irradiation-induced Self-organization $\quad 10$

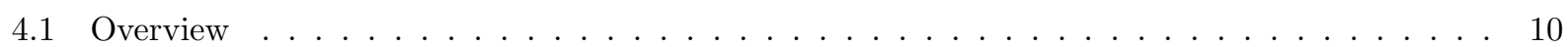

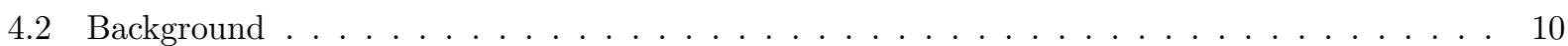

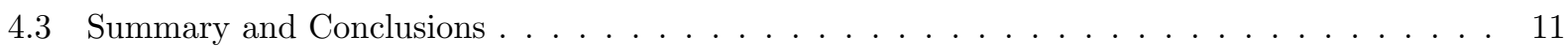

5 Publications Supported Fully or Partially by the Grant 13

6 Bibliography $\quad 16$ 


\section{Introduction and Background}

The main objective of this research was to develop new computational tools for in-service design and performance prediction of advanced fusion material systems by computational simulation. We also need to develop these computational tools to assist in planning and assessment of corresponding radiation experiments. In the following, we give a brief summary of the salient achievements of research supported by this grant.

\section{Radiation Hardening and Plastic Instabilities}

\section{$2.1 \quad$ Introduction.}

Metal deformation in response to external forces is well-established to be a result of dislocation generation and motion. While the onset of plastic yield is correlated with the initiation of dislocation motion, subsequent hardening or softening are clearly controlled by the continuation of such motion. Plastic yield and subsequent hardening (or softening) are primarily determined by the mechanisms that control dislocation motion. In unirradiated metals, dislocation-dislocation interactions play a very significant role in determining the characteristics of plastic deformation, where the impedance of dislocation motion is associated with the formation of sessile junctions or dipoles. In Body Centered Crystals (BCC), however, the flow stress and the presence of an upper yield point can be drastically changed by small additions of impurities. Also, in some alloyed FCC metals (e.g. copper crystals containing zinc), the upper yield point has also been observed. To explain this effect, Cottrell [9] showed that the flow stress at the upper yield point is a consequence of dislocation detrapping from impurity clouds, which are attracted to dislocations because of their elastic interaction with dislocations. It has thus long been recognized that interactions between dislocations and impurities, extrinsic and intrinsic lattice defects play a significant role in determining the mechanical properties of materials. In an irradiation environment, however, neutron collisions with lattice atoms produce copious densities of intrinsic defects, thus reducing or inhibiting dislocation motion in response to external forces. The initiation of plastic yield in irradiated metals is therefore almost entirely controlled by dislocation interaction with intrinsic defects. This situation is to be contrasted with the deformation characteristics of un-irradiated materials, where the flow stress and subsequent plastic work hardening are mainly determined by dislocation-dislocation interaction mechanisms.

The general features of the stress-strain curve of irradiated pure FCC metals are similar to those of unirradiated BCC metals containing impurities. At some critical irradiation dose (e.g. $\sim 0.1$ dpa in $\mathrm{Cu}$ ), an upper yield point emerges, followed by a drop in the yield strength. Most dislocations are observed to be heavily decorated by small, sessile interstitial clusters [29]. To explain the experimentally observed yield drop, and to understand the relationship between dislocation decoration and the yield behavior of irradiated materials, the Cascade Induced Source Hardening (CISH) model has been proposed (see references $[29,35,34])$. In this concept, Self Interstitial Atom (SIA) defect cluster mobility and trapping in the stress field of grown-in dislocations are assumed to be the main reason behind experimental observations of dislocation decoration, and the corresponding presence of an upper yield point in irradiated FCC metals and alloys. Decorations of dislocations in irradiated FCC metals indicate the presence of sessile interstitial dislocation loops produced by coalescence of mobile SIA clusters outside a stand-off distance from the dislocation core. Mobile SIA clusters that approach the dislocation at closer distances are absorbed into the dislocation core $[35,12]$. On the basis of this picture, the CISH model was used to estimate the flow stress of irradiated metals as the stress necessary to unlock dislocations from immobile SIA coagulated clusters. In this model, however, as in Kropua's analysis of similar hardening problems [18], dislocations are assumed to be rigid during their interaction with defects.

Once dislocations are released from localized SIA clusters, they will undoubtedly move very fast under the same applied stress that unlocked them, and unless their motion is further hindered, strain softening may occur. In irradiated FCC metals, dislocation motion past the initial yield point is determined by additional 
interactions with lattice defects in the form of sessile Stacking Fault Tetrahedra (SFTs) or Frank vacancy loops. On the other hand, it is found that a high density of microvoids in BCC metals control the postyield hardening or softening behavior in irradiated BCC metals [30]. The overriding theme here is that the phenomena of yield initiation, emergence of an upper yield point in FCC metals, the magnitude of radiation hardening (i.e. increase in CRSS), the subsequent hardening or softening, the formation of micro slip bands (i.e. dislocation channels), and eventual fracture of irradiated materials are all dictated by the details of dislocation-defect interactions. In companion overview articles [30, 23] address the relationships between dislocation-defect interaction and experimental observations on radiation hardening, yield drop, and postyield plasticity of irradiated BCC and FCC metals. The main objective of the present work, however, is to focus on the computational and mechanistic aspects of dislocation-defect interactions in irradiated materials. The two papers are therefore complementary, and are aimed at providing a comprehensive account of the major phenomena involved in determining plasticity and fracture of irradiated metals and alloys.

\subsection{Dislocation Unlocking from Cluster Atmospheres}

We present here results for the unlocking of dislocations from the collective elastic field of immobilized SIA cluster atmospheres, following the work of Ghoniem:01. A total of 15 SIA prismatic clusters, each with the same $\mathbf{b}=\frac{1}{2}[011], d=40, l=100$, and $y=80$, are shown to interact with an advancing dislocation on its glide plane. The external shear stress $\sigma_{11} / \mu$ or $\tau / \mu$ is gradually increased from 0 at intervals of $\Delta \tau / \mu=0.008 \%$. At each stage, the dislocation reaches a new stable configuration. The dislocation line becomes asymmetric, as interacts with the row of SIAs. When the normalized applied stress is increased to $0.112 \%$, the dislocation breaks away from one side of the cluster atmosphere. Once this process of unzipping begins, it is further accelerated by the change in local curvature.

The analytical results of Trinkaus:97a are also compared with the current numerical simulations. 15 equally-spaced clusters interact with a glide edge dislocation with two pinned ends, $d=40$, and $\mathbf{b}=\frac{1}{2}[\overline{1} 10]$ for both dislocation and clusters. The CRSS decreases with the increase in the stand-off distance, consistent with analytical estimates. Because of the short distance in between clusters, the dislocation breaks away in a rigid manner from its middle section. It is interesting to note that computer simulations show two distinct regimes: regime $\mathrm{A}(l<2 d)$, and regime $\mathrm{B}(l \geq 2 d)$. In regime $\mathrm{A}$, cluster fields overlap, and the force distribution on the segment does not vary significantly to allow dislocation buckling. Thus, the resistance to dislocation motion is controlled by the collective field of all clusters. In regime B, however, once the inter-cluster separation is larger than twice its diameter, the force variation of clusters over the segment is large, and the dislocation prefers to buckle in between them. The radius of curvature of this bulge is on the order of $\frac{1}{2}$ the inter-cluster distance. Thus, the resistance to dislocation motion becomes more local, where its radius of curvature determines the CRSS. A sudden jump is clearly observed when we cross from regime A to B.

\subsection{Dislocation Interaction with Sessile Defect Clusters}

A comprehensive computational algorithm has been developed to simulate dislocation interactions with dispersed barriers, which interact with dislocations with localized force fields that can be approximated as point forces. Nano-scale precipitates, Stacking Fault Tetrahedra (SFTs) and small voids are all sessile in irradiated materials, and one can assume that their force field is localized at a point on the glide plane. Because of the large density and the numerous interactions of such obstacles to dislocation motion, dislocation loops are parametrically represented as small circular arcs inscribed in between obstacle points on the glide plane. The general calculational algorithm works as described below.

The total number of obstacles (e.g. precipitates, vacancy clusters and SFTs) are calculated from their experimentally measured densities and the simulation cube size. The percentage of destructible obstacles (e.g. SFTs or vacancy clusters) is also specified. Each loop segment is represented by a circular arc, and its 
curvature is determined by the applied stress, sum of all interaction forces, and Burgers vector. When a loop segment encounters the nearest obstacle, it splits into two segments and each segment continues to move until it reaches its equilibrium curvature, or when the angle between the two tangents at the obstacle reaches a critical value, $\Phi_{c}$. A Kinetic Monte Carlo (KMC) procedure is implemented to determine the probability of obstacle destruction (for SFTs and microvoids), or the dislocation cutting through the obstacle (for precipitates). The probability of cutting/ destruction is calculated from the height of the energy barrier, the work done by the local forces at tangent points, and the lattice temperature. Once an event has been determined, the waiting time $t_{w}$ is calculated by the KMC method described in the previous section, and the obstacle is either destroyed or cut-through. After annihilation, these two segments merge into one and the unified segment is advanced till it meets the next obstacle on the plane, and the corresponding free flight time $t_{f}$ is also recorded. The total advance time between obstacles is the sum of these two, i.e. $t_{a}=t_{w}+t_{f}$. The procedure is repeated for all segments, and a general time clock records the corresponding time for the evolution of the dislocation loop shape.

When a vacancy cluster or microvoid is destroyed, the vacancy contents are assumed to be absorbed immediately into the dislocation core in between two obstacles, and a climb distance is obtained from the number of vacancies contained in the obstacle divided by the segment length. An expanding dislocation loop will then have segments on different, parallel glide planes. When the segment climbs and jumps to another parallel plane, the intersections between obstacles and the new plane are calculated and used for finding new interactions on that plane. The properties of every obstacle in 3D are tracked by array variables, which store integer numbers representing different properties. Once an obstacle (SFT or microvoid) is destroyed, it is immediately detected by tracking the corresponding array variable.

Periodic boundary conditions are implemented for the expansion of loops emanating from Frank-Read sources. The distance between each segment and the nearest boundary is calculated with every time step. Once a segment is detected to be out of the simulation boundaries, a different integer is assigned to the array variables, and the segment motion is continued from the boundary at the opposite side, and the segment is placed on a parallel glide plane. The distance between any two segments from the same dislocation loop (FR-source) is calculated to determine if it is less than a prescribed length (about the half of average segment length). The two closest segments are then determined, and annihilation is implemented. The old loop will then be split at these two segments and re-connected to the segment on the other side. This process involves the generation of a new open loop with two pinned ends (F-R source), and the majority of the old loop becomes an expanding closed loop. All properties from the old loop are also assigned to the new open loop in order that it can continue its motion. The interaction forces between segments are calculated every time step because they are used to determine the segment curvature. The free flight time $t_{f}$ for a segment is measured from an initial straight segment in between two obstacles to the curved configuration, where the segment touches the nearest obstacle. Therefore, the free flight time is determined by the curvature and segment length. The waiting time is measured from the time an obstacle is contacted till its cutting or annihilation.

\subsection{Interaction with Stacking Fault Tetrahedra (SFTs) in FCC Metals}

Once dislocations are freed from their locking defect cluster atmospheres, they will move on their glide planes till they encounter other obstacles to their motion. The yield drop at this moment is associated with a sudden increase in the plastic strain originating from released dislocations, thus requiring a drop in the applied stress to maintain a constant rate of total strain. The post yield behavior of irradiated FCC specimens is determined by dislocation interaction with sessile vacancy clusters, which can be either SFT type, or vacancy loop type. Here, we consider the case of irradiated annealed copper at room temperature. The experimentally-measured values of SFT density and size are given in Table (1) below.

The resistance of SFTs to the expansion of dislocations from F-R sources requires an increase in the applied stress to maintain a constant rate of strain. Thus, on active glide planes, a local stress-strain diagram can be obtained, where the results are shown for four different irradiation doses. The macroscopic 
Table 1: Experimentally-measured SFT density, size and hardening $\left(\Delta \sigma_{\text {exp }}\right)$ in annealed $\mathrm{Cu}$, and calculated results for different critical angles

$[27]$

\begin{tabular}{|c|c|c|c|c|c|c|c|}
\hline Case & Dose & $\begin{array}{l}\Delta \sigma_{\text {exp }} \\
(\mathrm{MPa})\end{array}$ & $\begin{array}{c}\text { SFT Density } \\
\left(\times 10^{-23} M^{-3}\right)\end{array}$ & $\begin{array}{l}\text { SFT Size } \\
(\mathrm{nm})\end{array}$ & $\Phi_{c}=160^{\circ}$ & $\begin{array}{c}\text { Calculated } \Delta \sigma \\
\Phi_{c}=165^{\circ}\end{array}$ & $\Phi_{c}=170^{\circ}$ \\
\hline 1 & 0.01 & 38 & 0.45 & 3.0 & 71 & 39 & 31 \\
\hline 2 & 0.1 & 105 & 1.4 & 4.5 & 120 & 105 & 80 \\
\hline 3 & 0.2 & 115 & 1.7 & 4.5 & 145 & 115 & 100 \\
\hline 4 & 0.3 & 120 & 2.5 & 4.0 & 155 & 125 & 110 \\
\hline
\end{tabular}

stress-strain curve, however, cannot be precisely determined unless one knows the fraction of active slip volumes within the tested sample; a task beyond the capabilities of current simulations. Following the yield drop, the lower yield point can be predicted by computer simulations for the interaction between released dislocations and SFTs that intersect glide planes. The only adjustable parameter in these calculations is the critical angle for destruction of a single SFT, which is found to be $\Phi_{c}=165^{\circ}$ for optimum correspondence between experiment and computer simulations. Table (1) shows the sensitivity of the results of computer simulations to variations in the value of the critical angle, $\Phi_{c}$.

\subsection{Interaction with Microvoids in BCC Metals}

In irradiated BCC metals, vacancies generated by collision cascades form small voids, while interstitials are carried away from cascade centers in SIA clusters. Therefore, dislocations that are released from SIA cluster atmospheres will interact with dispersed voids in the matrix in a similar fashion to the situation in FCC metals, where dislocations interact with SFTs or vacancy loops. Under the action of an applied stress, dislocations impinge on nano-size voids and may destroy them if the work done by local forces exerted by dislocations on microvoids exceeds a critical value determined by the elastic interaction energy. In the present computer simulations, we assume that the small nano-size void is destroyed by the dislocation, once the angle between the arms of the dislocation that surround the void exceeds a critical value, $\Phi_{c}$. Thus, $\Phi_{c}$ is viewed as the only adjustable parameter here, and its value can be precisely calculated through Molecular Dynamics (MD) computer simulations [23]. It is important, however, to determine the energy of dislocation cutting through voids rather than $\Phi_{c}$ in MD simulations, and then convert that energy into the corresponding $\Phi_{c}$ by the elastic point force model.

The model parameters are given in Table(2) below. Initially, dislocations in a local area of stress concentration or statistically-low SIA cluster atmosphere density are activated when the local shear stress reaches a critical value. One such F-R source is activated at a stress level of $30 \mathrm{MPa}$. When the applied stress is increased to $65 \mathrm{MPa}$, several F-R sources are shown to have their dislocations bowing out in response to the applied stress and mutual interaction forces. However, only a few have expanded significantly to reach the edge of the simulation cube. Also shown in the same figure is the pre-annihilation stage of one of the F-R source dislocations, where two segments are about to annihilate, thus creating a full loop and restoring the initial pinned dislocation segment of the source. Further activation of dormant F-R sources is achieved, when the local microvoid density is effectively reduced by the passage of nearby dislocations. A domino effect is thus created, where the destruction of microvoids in a local region by one source activates other nearby sources. It is to be noted that the dislocation loop structure is not planar, because of the continuous climb process associated with each dislocation glide event. At a stress level of $70 \mathrm{MPa}$, dislocation loops start to impinge on the simulation box boundary, and at that point, periodic boundary conditions are implemented to inject those loop segments that emerge from one side of the boundary to the other side, as can be seen in FIG.(??). The evolution process is terminated when the leading-edge loop reaches the grain boundary or 
surface (which is assumed here to be $10 \mu \mathrm{m}$ ), and successive dislocation loops interact with one another to form a non-planar pileup of loops that would exert sufficient back stress on all sources to shut them off. The structure of the evolving non-planar pileup of loops is shown from the side view (the $<0 \overline{1} 0>$-direction is towards the viewer) in FIG. (??).

Table 2: Input parameters for the computer simulations of FIG.(1) for irradiated Fe.

\begin{tabular}{|ccccccc|}
\hline \hline $\begin{array}{c}\text { Dose } \\
(\mathrm{dpa})\end{array}$ & $\begin{array}{c}\sigma_{\exp } \\
(\mathrm{MPa})\end{array}$ & $\begin{array}{c}\text { Void Density } \\
\left(\times 10^{-23} M^{-3}\right)\end{array}$ & $\begin{array}{c}\text { Void Size } \\
(\mathrm{nm})\end{array}$ & $\begin{array}{c}\text { Simulation Cube } \\
\text { Size }(\mu \mathrm{m})\end{array}$ & $\begin{array}{c}\text { Number of } \\
\text { F-R sources }\end{array}$ & $\begin{array}{c}\text { Grain Size } \\
(\mu \mathrm{m})\end{array}$ \\
\hline 0.01 & 65 & 0.5 & 0.6 & 2.6 & 10 & 10 \\
\hline
\end{tabular}

\subsection{Conclusions and Future Outlook}

The main theme of the present overview is to delineate how large-scale computer simulations of dislocationdefect interactions in irradiated materials can reveal physical insights that can be used to construct either simpler analytical models, or help in experimental design. The ultimate objective of computer simulations is to be useful in designing radiation-resistant alloys, once the basic mechanisms are clearly determined. This task is not complete, however, unless the results of computer simulations are compared to specific experiments that are designed to test the validity of the mechanisms themselves. In this article, we've shown that largescale computer simulations provide insight that cannot be gained from analytical theory or experiments alone, but once all aspects are considered, one is more certain of the nature of investigated mechanisms. In a companion article ([30]), the emphasis is shifted towards the question of how one can use experimental observations to drive more realistic computer models. We conclude here that the current methods of computer simulations of dislocation defect interactions, namely, the DD and KMC techniques used separately and in combination, have resulted in defining a clearer picture of how SIA clusters attract to dislocations to decorate and lock them in place, how they form rafts and how they pin themselves and not move around at vast speeds. It also resulted in understanding the fundamental mechanism of dislocation unlocking from defect cluster atmospheres, where dislocations pull themselves out of such atmospheres by reconfiguration and shape instabilities. Finally, the basic mechanisms of radiation hardening beyond the upper yield point, and the ensuing nucleation and propagation of dislocation channels have been demonstrated by large-scale computer simulations. The agreement with experimental observations of radiation hardening has been demonstrated with only one adjustable parameter. In addition, correspondence between TEM observations on dislocation decoration, raft formation and dimensions of dislocation channels has been shown, and is fully discussed in reference $([30])$.

Future efforts are expected to address a number of yet un-resolved issues in the area of radiation embrittlement and plastic instabilities. These are:

1. Computer simulations of entire grains, coupling DD with the more macroscopic crystal plasticity models, where lattice rotations, load transfer, and dislocation interaction with boundaries may determine the spacings observed in between dislocation channels;

2. An emphasis on materials design aspects, where one would integrate computer models of alloyed materials with experimental design to understand how plastic flow can be managed over larger volumes to delay or retard dislocation channel formation and fracture;

3. Utilization of computer simulations to design new experiments, which would reveal the synergistic effects of applied stress and irradiation on material deformation. 

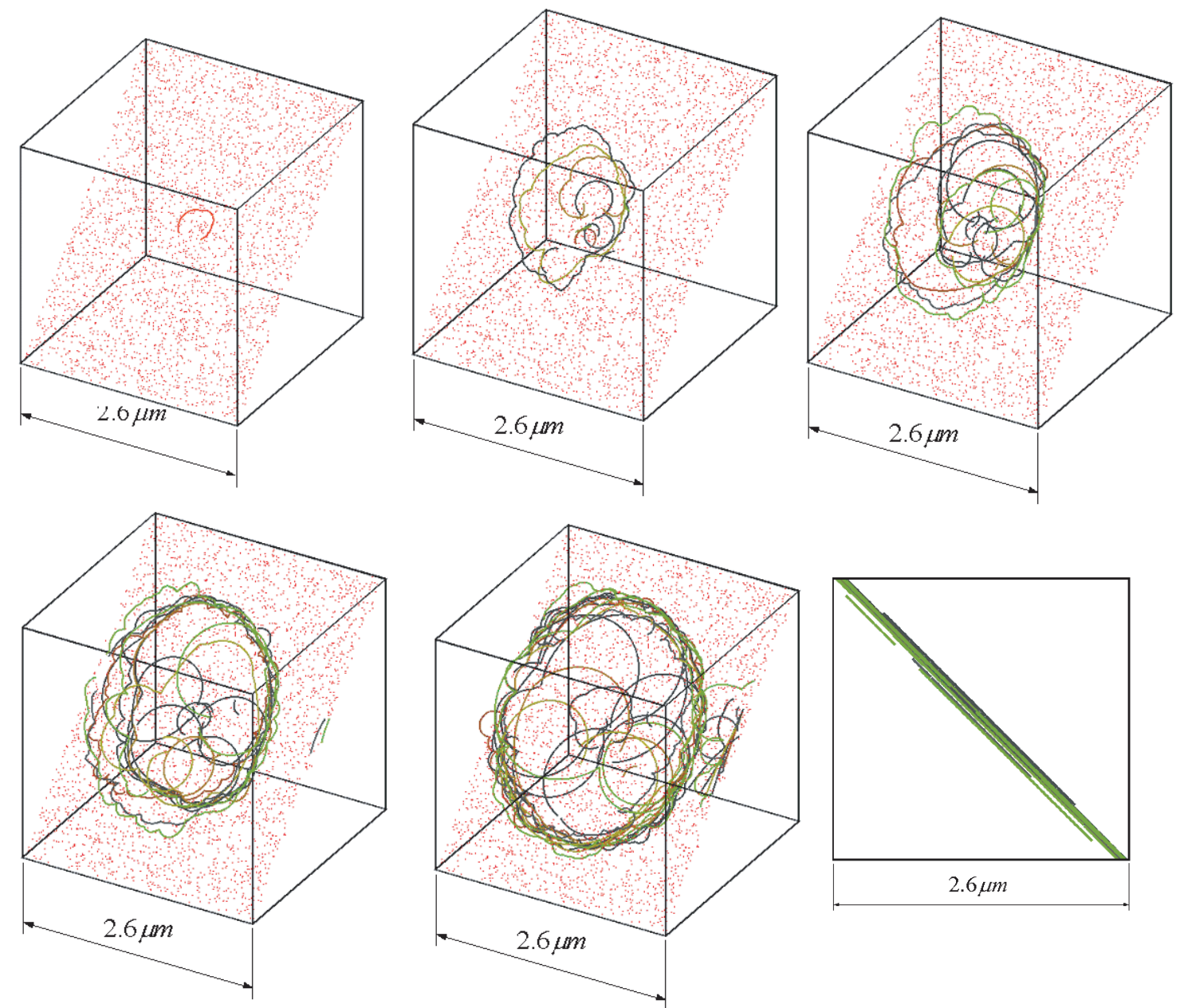

Figure 1: Various stages for the development of a dislocation channel in irradiated Fe. Figures (a-e) show the evolution of dislocation loops as they interact amongst themselves and microvoids on planes parallel to $\{101\}$-planes, while Figure(f) shows the dislocation channel edge-on (the $<0 \overline{1} 0>$-direction is towards the viewer 


\section{Non-equilibrium Phase Transitions}

\subsection{Overview of Achievements}

The Principal investigator supervised the thesis of Marios Demetriou, which had the objective of developing kinetic models for the non-equilibrium formation of tugsten carbides during rapid heating and solidification. The work resulted in the publication of a number of papers, and the establishment of a kinetic methodology for determination of the phase fraction of non-equilibrium carbides. This work can be readily extended to the irradiation conditions to treat the formation of carbides, nitrides and oxides. In the following, we outline some of the model highlights.

A dynamic computational model developed within the context of the classical theory of phase evolution has been developed and applied to the W-C system to simulate the kinetics of graphite nucleation during non-equilibrium peritectic melting of WC. The kinetic variables used in the model are obtained directly from the free energy formulations that characterize the stable and metastable equilibria between WC, liquid, and graphite. The isothermal kinetic analysis suggests that transformation time decreases monotonically with increasing superheat such that the minimum transformation time occurs at the metastable congruent melting point of WC $(\sim 3107 \mathrm{~K})$. To crystallize 1-ppm of graphite, the minimum transformation time is computed to be 2 ns. The non-isothermal kinetic analysis suggests that under moderate to high heating rates $\left(10^{4}-10^{6} \mathrm{~K} / \mathrm{s}\right)$ graphitization is completed at superheats of $40-50 \mathrm{~K}$, while under ultra-high heating rates $\left(\sim 10^{8} \mathrm{~K} / \mathrm{s}\right)$ graphitization remains incomplete giving rise to metastable congruent melting of WC.

In this study, a computational model is developed to simulate the kinetics of graphite nucleation upon WC superheating. The aim is to assess the importance of nucleation kinetics in limiting the rate of peritectic melting reaction. This study constitutes the first attempt to model nucleation kinetics in peritectic melting. The present model, which was developed in the context of classical nucleation theory, simulates the stochastic process of crystal nucleation by modeling the dynamics of cluster evolution. The model also accounts for size-dependent growth of finite size nuclei. The kinetic variables used in the model were obtained directly from the free energy formulations that characterize the stable and metastable equilibria between WC, liquid, and graphite. The empirical relations that govern kinetic properties under conditions of supercooling are adopted in the present model and are extrapolated above equilibrium to approximate properties under superheating conditions. Furthermore de-carburization was neglected and fixed composition at 50\%-C was assumed throughout the process. The model was applied to investigate the kinetics of graphite nucleation via the stable peritectic reaction $W C \rightarrow$ liq + gra upon annealing or continuous heating, and to examine the possibility of complete kinetic bypassing of the graphite phase.

\subsection{Publications on Phase Evolution}

The following publications resulted form the thesis of Marios Demetriou on non-equilibrium phase transformations:

1. Marios D. Demetriou, Nasr M. Ghoniem, Adrienne S. Lavine, "Kinetic Modeling of Phase Selection during Non-Equilibrium Solidification of WC," Acta Materialia, 50 (6):1421-1432 (2002).

2. Marios D. Demetriou, Nasr M. Ghoniem, Adrienne S. Lavine, "Effects of Nucleation Transience on the Kinetics of Crystallization," J Chem Phys, 117:23 (2002).

3. Marios D. Demetriou, Nasr M. Ghoniem, Adrienne S. Lavine, "Computation of Metastable Phases in Tungsten-Carbon System," Journal of Phase Equilibria, 23(4):305-309, (2002).

4. Marios D. Demetriou, Nasr M. Ghoniem, Adrienne S. Lavine, "Modeling of Graphitization Kinetics during Peritectic Melting of Tungsten Carbide," Acta Materialia, in press, (2002). 


\section{Irradiation-induced Self-organization}

\section{$4.1 \quad$ Overview}

Irradiation of materials by energetic particles (e.g. electrons, ions and neutrons) is associated with very high internal power dissipation, which can drive the underlying nano- and microstructure far from normal equilibrium conditions. One of the most unusual responses in this connection is the ability of the material's nano- and microstructure to self-assemble in well-organized, two- and three-dimensional periodic arrangements. We reviewed and assessed experimental evidence and theoretical models pertaining to the physical understanding of nano- and microstructure self-organization under irradiation conditions. Experimental observations on the formation of self-organized defect clusters, dislocation loops, voids and bubbles were presented and critically assessed. Implantation of metals with energetic helium results in remarkable selfassembled bubble super-lattices with wavelengths (super-lattice parameters) in the range of 5-8 nm. Ion and neutron irradiation produce a wide variety of self-assembled 3-D defect walls and void lattices, with wavelengths that can be tailored in the range of 10's to 100's of nanometers. Theoretical models aimed at explaining these observations were introduced, and a consistent description of many features is outlined. The primary focus of the most recent modeling efforts, which are based on stability theory and concepts of non-linear dynamics, was to determine criteria for the evolution and spatial symmetry of self-organized microstructures. The correspondence between this theoretical framework and experimental observations was also examined, highlighting areas of agreement and pointing out unresolved questions.

\subsection{Background}

The phenomena of pattern formation and self-organization have been viewed as natural responses of complex systems to strong external stimulation. The collective interaction between system components under external driving forces that drive the system far from equilibrium results in the self-organization of its constituents (e.g. [22], [10]). Some of the salient successes of this viewpoint can be found in the behavior of chemical (e.g. [5], [2]), liquid crystal nematics (e.g. [4], [24] ), and fluid systems (e.g. [8], [7]). It has also been recognized that condensed matter systems show a rich variety of patterns and self-assembled microstructures under conditions as diverse as solidification [31], electro-chemical deposition [20], plastic deformation (e.g. [21], [17]), surface modifications [16], rapid laser heating [3], irradiation by energetic particles [11], magnetic domains [26], and more recently, atom deposition into self-assembled mono-layers [41], [33]. The particular situation of energetic particle irradiation is quite interesting, and we plan to focus our attention in this article on reviewing the current understanding of the underlying physical mechanisms. The existence of an extensive experimental database in this area, and the concurrent evolution of contemporary theoretical frameworks allow us to present a broad perspective on the physical nature of self-organization in irradiated materials.

During the last three decades, numerous experimental observations on irradiated materials have systematically demonstrated the existence of fully or partially ordered nano- and microstructure in materials under energetic particle irradiation. The phenomenon appears to be of a general nature, and not confined to one type of microstructure. Voids, precipitates, vacancy clusters, Stacking Fault Tetrahedra (SFT's), gas bubbles and Self-Interstitial-Atom (SIA) clusters have all been observed to be spatially arranged in nano-scale, selfassembled patterns of typical dimensions 2-3 orders of magnitude greater than the atomic spacing. Striking observations have shown that in some cases, complete spatial isomorphism exists between the periodic structure of defect distributions and that of the fundamental atomic lattice. These experimental observations are particularly true for the spatial ordering of bubble and void microstructures in irradiated materials [25], [39]. Singh and Evans [28] have recently reviewed the experimental results obtained on defect production and accumulation in irradiated materials, and emphasized the systematic differences between void and defect clusters in FCC and BCC metals and alloys. An important characteristic of void and bubble lattices is their spatial orientation along crystallographic directions, at least for the three main metal structures (FCC, 
BCC, and HCP). Jäger and Trinkaus reviewed the experimental observations of ordered defect structures in irradiated materials, and concluded that the isomorphy of the ordered defect structures with the host lattice is due to elastic interactions in the case of defect walls and anisotropic defect transport in the case of bubble and void superlattices [15].

The implications of understanding the physical nature of nano- and microstructure self-organization are quite clear from a technological perspective. The relationships between the material's microstructure and its mechanical and physical characteristics are established by a combination of experimental, empirical and theoretical methods. It is expected, therefore, that a physical understanding of the nano- and microstructure and its dependence on irradiation and material conditions would lead to better approaches to the design of radiation-resistant materials in nuclear technologies (e.g. fission and fusion energy). At the same time, ion, plasma and electron beam processing are becoming valuable tools in the manufacturing of electronic, photonic and microelectromechanical (MEMS) devices. While the impact of a physical understanding of nano- and microstructure self-organization phenomena on the variety of technologies discussed here is difficult to assess, the intrinsic scientific value is quite valuable. The collective behavior of constituents in hydrodynamic, chemical and liquid crystal systems has been systematically shown to result in pattern formation driven by dynamical instabilities. In the solid state, however, few examples exist, where the link between dynamical instabilities and the evolution of a self-organized nano- and microstructure has been clearly established. For example, while self-organized dislocation microstructure has been widely observed under cyclic and monotonic deformation conditions (e.g. Persistent Slip Bands, dislocation arrays, cells and subgrains), the reasons for its emergence have not yet been established [1].

Concepts on the behavior of dynamical systems have been successfully applied to the problem of microstructure evolution in irradiated materials. Self-organization of irradiated microstructures can thus be viewed as one of the few examples in the solid state, where patterning can be directly linked to the collective dynamical behavior of constituent defects. The aim of this work was to review current models of self-organized microstructure in irradiated materials, and compare the theoretical findings with experimental observations.

\subsection{Summary and Conclusions}

One may conclude that a comprehensive theoretical framework for the analysis of spatial instabilities in the nano- and microstructure of irradiated materials has now been obtained. This framework, which was initiated over two decades ago, results from the succession of works based on a well-established rate theory of microstructure evolution under irradiation. The theory has been pursued both qualitatively and numerically to ascertain the main processes leading to nano-structure pattern selection in irradiated materials. Throughout this review, we emphasized theoretical conditions, which are consistent with experimental observations, for the development of spatially organized (or self-assembled) nano-structures. Some areas which still lack clear understanding include the effects of void density on the magnitude of the selected wavelength, the smaller wavelength of bubble lattices as compared to void lattices, and the unique conditions that lead to loop patterning in $\mathrm{Cu}$.

The rate theory kinetic models described here incorporate basic elements of defect generation, interactions and mobility. They also allow an explicit determination of the instability threshold and its dependence on material and irradiation parameters. Determination of selected nano-structure and its subsequent evolution requires post-bifurcation analysis. Such analysis may be performed analytically and numerically [36]. In the weakly non-linear regime, the analysis is based on a derivation of density perturbations for the Fourier components of immobile defects, by invoking a quasi-static approximation. With this approximation, Ginzburg-Landau type amplitude equations for the nano-structure may be derived, with slowly varying time-(or dose-) dependent coefficients. Generic conclusions of pattern selection theory are then recovered, showing non equilibrium phase transitions leading to the eventual formation of wall defect structures, with a wavelength that decreases with the irradiation dose. Furthermore, the nano-structure is expected to be extremely sensitive to even small anisotropies. In particular it should have parallel orientations with the 
directions of high interstitial mobility, as is experimentally observed for vacancy cluster walls, and for void and bubble super-lattices as well.

The results of the theory are in general agreement with the majority of experimental observations. Most of the qualitative features of self-assembled nano-structures can be explained on the basis of the present level of theoretical development. Theory predictions for the gradual transition from spatially random to self-assembled nano-structure, the insensitivity of the selected wavelength to temperature and its weak dependence on dose rate, the isomorphism between the nano-structure super-lattice and the underlying crystal, the decrease of observed wavelength with dose and the zig-zag patterns of vacancy clusters are all in qualitative agreement with experimental observations. Surprisingly good quantitative agreement between theory and experiment is obtained for the phase boundaries between ordered and random nano-structures, and for the magnitude of the selected pattern wavelength. On the other hand, the apparent experimental difficulty in verifying the exact mechanism responsible for pattern formation in irradiated copper is somewhat unresolved. While Jäger clearly showed well-defined self-assembled vacancy clusters in copper, the majority of other experimental results did not show pattern formation. To resolve the fundamental differences that lead to such unusual on/off condition within the framework of the current theory require additional specifically planned experiments.

In summary, the necessary ingredients for the kinetic rate theory model to be consistent with experimental observations are three. These are :

1. An excess bias of dislocations towards interstitial atom absorption ;

2. A fraction of vacancies to be produced directly in clusters, as a result of collision cascades;

3. A small degree of interstitial diffusional anisotropy.

The production asymmetry (so called production bias) appears not to be essential to triggering microstructure instabilities (i.e. $\varepsilon_{i}=0$ ), as long as a small fraction of vacancies is produced in clustered sessile configurations. However, if sessile SIA clusters are also produced in collision cascades (i.e. $\varepsilon_{i} \neq 0$ ), their presence combines to accelerate the onset of spatial instabilities. Comparison between theory predictions and experimental data is shown in Figure (??). The data include Ni+ ion [19, 6, 37]-[38], fission neutron $[32,40]$, and proton $[14,13]$ irradiations. The filled symbols denote conditions where defect cluster wall formation was observed. 


\section{$5 \quad$ Publications Supported Fully or Partially by the Grant}

1. N.M. Ghoniem, H. Heinisch H. Huang, L. Kubin, Yu, and S. Yip, Guest Editors, Special Issue "Multiscale Materials Modeling," J. Comp.-Aided Mater. Design, 6, No. 2\&3 (1999) 374 pages.

2. N.M. Ghoniem, "Clustering Theory of Atomic Defects," Radiation Effects and Defects in Solids, 148: 269-318 (1999).

3. H. Huang, N.M. Ghoniem, T. Diaz de la Rubia, M. Rhee, H. Zbib and J. Hirth, "Stability Of Dislocation Short-Range Reactions In BCC Crystals," J. Eng. Mat. \& Tech., 121(2): 143 (1999).

4. N.M. Ghoniem, "Curved Parametric Segments For The Stress Field Of 3-D Dislocation Loops," J. Eng. Mat. \& Tech., 121(2): 136 (1999).

5. N. M. Ghoniem, L. Sun, "Fast Sum Method for the Elastic Field of 3-D Dislocation Ensembles," Phys. Rev. B, 60(1): 128-140 (1999).

6. L. J. Perkins, B. G. Logan, M.D. Rosen, M.D. Perry, T. Diaz de la Rubia, N.M. Ghoniem, T. Ditmire, S.Wilkes and P.T.Springer, "The Investigation of High-Intensity-Laser Driven Micro Neutron Sources for Fusion Materials Applications at High Fluence," Nuclear Fusion., 40(1):1-19 (2000).

7. N.M. Ghoniem, B. N. Singh, L. Z. Sun, and T. Diaz de la Rubia, "Interaction and Accumulation of Glissile defect Clusters Near Dislocations," J. Nucl. Mater, 276: 166-177 (2000).

8. N.M. Ghoniem, S.- H. Tong, and L. Z. Sun , "Parametric Dislocation Dynamics: A Thermodynamicsbased Approach to Investigations of Mesoscopic Plastic Deformation," Phys. Rev. B, 139(1): 913-927 (2000).

9. L. Sun, N.M. Ghoniem, and B.N. Singh, "3-D Dislocation Dynamics Study of Plastic Instability In Irradiated Copper," J. Nucl. Mater., 283:741(2000).

10. S. Sharafat and N.M. Ghoniem, "Comparison of A Microstructure Evolution Model With Experiments on Irradiated Vanadium", J. Nucl. Mater.,283:789(2000).

11. S.J. Zinkle and N.M. Ghoniem, "Operating Temperature Windows for Fusion Reactor Structural Materials", Fusion Engineering and Design,51-52:55(2000).

12. S. Sharafat, M. Demetriou , N. Ghoniem , B. Williams, R. Nygren, "Enhanced surface heat removal using a porous tungsten heat exchanger," Fus. Tech. 39(2):863-867, Part 2, 2001.

13. L.Z. Sun, N.M. Ghoniem, and Z.Q. Wang, "Analytical and Numerical Determination of the Elastic Interaction Energy between Glissile Dislocations and Stacking Fault Tetrahedra in FCC Metals", J. Mat. Sci. \& Engr., A309-310:178183 (2001).

14. Nasr M. Ghoniem, "Radiation Damage Correlations For Fusion Conditions", Encyclopedia of Materials: Science and Technology, Pergamon Press, Elsevier Science Publication, 3413-3418 (2001), ISBN:0-080431526 .

15. N.M. Ghoniem, S.H. Tong, B.N. Singh, and L.Z. Sun, "On Dislocation Interaction with Radiationinduced Defect Clusters and Plastic Flow Localization in FCC Metals", Phil. Mag. A, 81 (11): 2743-2764 (2001).

16. G.R. Odette, B.D. Wirth, D. J. Bacon and N.M. Ghoniem, "Multi-scale, Multi-physics Modeling of Radiation-damaged Materials: Embrittlement of Pressure Vessel Steels", MRS Bulletin, 176-81(2001).

17. N.M. Ghoniem, J.M. Huang, and Z.Q. Wang, "Affine Covariant-contravariant Vector Forms for the Elastic Field of Parametric Dislocations in Isotropic Crystals," Phil. Mag. Lett., 82(2): 55-63 (2002). 
18. N.M. Ghoniem and J.M. Huang, "Computer Simulations of Mesoscopic Plastic Deformation with Differential Geometric Forms for the Elastic Field of Parametric Dislocations: Review of Recent Progress,"Invited Paper at the 5th Euro-Conference on Mechanics of Materials, Delft, Netherlands, March 6-9, 2001, Also J. de Physique IV, 11(5):53-60 (2001).

19. N.M. Ghoniem,D. Walgraef and S. J. Zinkle, "Theory and Experiment of Nanostructure Self-organization in Irradiated Materials,"Invited Perspective Article, J. Comp. Aided Mat. Design, 8:1-38 (2002).

20. Jianming Huang and Nasr M. Ghoniem, "The Dynamics of Dislocation Interaction with Sessile SelfInterstitial Atom(SIA) Defect Cluster Atmospheres,", J. Comp. Mat. Science,23:225234 (2002).

21. R. Martinez and N. M. Ghoniem, "The Infuence of Crystal Surfaces on Dislocation Interactions in Mesoscopic Plasticity: A Combined Dislocation Dynamics- Finite Element Approach,", J. Comp. Meth. Engr. Science, CMES, 3(2):229-243 (2002).

22. Nasr M. Ghoniem and Kyeongjae Cho, "The Emerging Role of Multiscale Modeling in Nano- and Micro-mechanics of Materials," J. Comp. Meth. Engr. Science, CMES, 3(2):147-173 (2002).

23. N. M. Ghoniem, S.H. Tong, J. Huang, B.N. Singh, and M. Wen, "Mechanisms of Dislocation-Defect Interactions in Irradiated Metals Investigated by Computer Simulations," J. Nucl. Mater., 307311: 843851 (2002).

24. B.N. Singh, N.M. Ghoniem and H. Trinkaus, "Experiment-Based Modelling of Hardening and Localized Plasticity in Metals Irradiated Under Cascade Damage Conditions," J. Nucl. Mater., 307311:159170 (2002).

25. Marios D. Demetriou, Nasr M. Ghoniem, Adrienne S. Lavine, "Kinetic Modeling of Phase Selection during Non-Equilibrium Solidification of WC," Acta Materialia, 50 (6):1421-1432 (2002).

26. Marios D. Demetriou, Nasr M. Ghoniem, Adrienne S. Lavine, "Effects of Nucleation Transience on the Kinetics of Crystallization," J Chem Phys, 117:23 (2002).

27. Marios D. Demetriou, Nasr M. Ghoniem, Adrienne S. Lavine, "Computation of Metastable Phases in Tungsten-Carbon System," Journal of Phase Equilibria, 23(4):305-309, (2002).

28. Marios D. Demetriou, Nasr M. Ghoniem, Adrienne S. Lavine, "Modeling of Graphitization Kinetics during Peritectic Melting of Tungsten Carbide," Acta Materialia, in press, (2002).

29. X. Han, N.M. Ghoniem and Z. Wang, "Parametric Dislocation Dynamics of Anisotropic Crystalline Materials," Phil. Mag. A., in press, (2002).

30. D.Walgraef and N.M.Ghoniem, "The Effects of Glissile Interstitial Clusters on Microstructure Selforganization in Irradiated Materials," Phys. Rev. B, in Press, (2002).

31. Nasr M. Ghoniem, Hanchen Huang, Esteban Busso, and Nicolas Kioussis, "Multiscale Modeling of Nano- and Micro-Mechanics: an Overview," Phil. Mag. A, in press, (2002).

32. Jianming Huang and Nasr M. Ghoniem, "Accuracy \& Convergence of Parametric Dislocation Dynamics (PDD)," Mod. Sim. Mat. Sci. Engr., 10:1-19 (2002).

33. N.M. Ghoniem, and B.N. Singh, "Dislocation Dynamics Study of the onset of Plastic Instabilities in Irradiated Materials,"Proc. of 20th RISO International Symposium on Materials Science, DeformationInduced Microstructures: Analysis and Relationship to Properties, Bilde-Sorensen, et al., Eds., September 6-10, 1999, Roskilde, Denmark, page 41-60.

34. C.P.C. Wong, R.E. Nygren, C.B. Baxi, P. Fogarty, N.M. Ghoniem, H. Khater, K. McCarthy, B. Merril, B. Nelson, E. Reis, S. Sharafat, R. Schleicher, D.K. Sze, M. Ulrickson, S. Williams, M. Youssef, and S. Zinkle, "Helium-cooled Refractory Alloy First Wall and blanket Evaluation,"Proc. of the fifth International Symp. On Fusion Nuclear Technology (ISFNT-5), September 1999, Rome, Italy. 
35. Nasr M. Ghoniem, "Computational Methods For Mesoscopic, Inhomogeneous Plastic Deformation", Proceedings of First Latin American Symposium on Materials Instabilities, Valpareso, Chile, Kluwer Publication, 2000. 


\section{Bibliography}

\section{References}

[1] R.J. Amodeo and N. M. Ghoniem. A review of experimental observations and theoretical models of dislocation cells. Res Mech., 23:137, 1988.

[2] F. Baras and D. Walgraef. Physica, A188, 1992.

[3] D. Bauerle. Laser Processing and Chemistry. Springer-Verlag, New York, 1996.

[4] Zimmermann W. Bodenschatz, E. and L. Kramer. J.Phys., 49, 1988.

[5] Borckmans, G. Dewel, A. De Wit, and D. Walgraef. Turing bifurcations and pattern selection. Chemical Waves and Patterns, Kapral R., and Showalter K. (Eds.), Kluwer, Dordrecht:323, 1994.

[6] J.L. Brimhall. Battelle pacific northwest laboratory report. Technical Report BNWL-1839, 1974.

[7] F. H. Busse and N. Riahi. J. Fluid Mech., 96:243, 1980.

[8] F. H. Busse. Rep. Prog. Phys., 41:1929, 1978.

[9] A.H. Cottrell. Report of conference on the strength of solids(university of bristol), england, physical society, london. 30:30, 1948.

[10] M. C. Cross and P. C Hohenberg. Rev. Mod. Phys., 65:851, 1993.

[11] J.H. Evans. Nature, 29:403, 1971.

[12] N.M. Ghoniem, B.N. Singh, L.Z. Sun, and T. Diaz de la Rubia. Interaction and accumulation of glissile defect clusters near dislocations. J. Nucl. Mater., 276:166, 2000.

[13] Ehrhart P. Jäger, W. and W. Schilling. Solid State Phenomena, 3-4:297, 1988.

[14] W. Jäger and et. al. Mater. Sci. Forum, 15-18:887, 1987.

[15] W. Jäger and H. Trinkaus. J. Nucl. Mater., 205:394, 1993.

[16] Singhal S.C. Kossowsky, R. Surface Engineering, Surface Modification of Materials. Dordrecht, 1984.

[17] J. Kratochvil. Rev.Phys.Appliquée, 23:419, 1988.

[18] F. Kroupa. Czech. J. Phys., B10:284, 1960.

[19] G.L. Kulcinski and Brimhall J.L. Effects of radiation on substructure and mechanical properties of metals and alloys. 1973.

[20] M.Q. López-Salvans, F. Sagués, and J. Claret. Fingering in thin layer electrodeposition. the Proceedings of the 1st Latin American School on Materials Instabilities, held in Valparaiso, Chile, Dec 98, Kluwer ublication, 2000.

[21] H. Neuhauser. Plastic instabilities and the deformation of metals. Patterns, Defects and Materials Instabilities, Walgraef, D. and Ghoniem, N.M. (Eds.), Kluwer Academic Publishers,Dordrecht, 1990.

[22] G. Nicolis, G. Dewel, and J. Turner. Order and Fluctuations in Equilibrium and Nonequilibrium Statistical Mechanics. Wiley Publishers, New York, 1981.

[23] Yu. N. Osetsky, D.J. Bacon, B.N. Singh, and B. Wirth. Atomistic study of generation, interaction, accumulation and annihilation of cascade-induced defect clusters. These proceedings, 2002. 
[24] I. Rehberg, B.L. Winkler, M. de la Torre Juarez, S. Rasenat, and W. Schopf. Festkorperprobleme/Advances in Solid State Physics, 29:35, 1989.

[25] S. Sass and B.L. Eyre. Philos. Mag., 27:1447, 1973.

[26] M. Seul and R. Wolfe. Phys. Rev. A, 46:7534, 1992.

[27] B.N. Singh, D.J. Edwards, and P. Toft. J. Nucl. Mat., 299:205, 2001.

[28] B.N. Singh and J.H. Evans. J. Nucl. Mat., 226:277, 1995.

[29] B.N. Singh, A.J.E. Foreman, and H. Trinkaus. J. Nucl. Mater., 249:103, 1997.

[30] B.N. Singh, N.M. Ghoniem, and H. Trinkaus. Experiment-based modeling of hardening and localized plasticity in metals irradiated under cascade damage conditions. Thesis proceedings, 2002.

[31] G. I. Sivashinsky. Physica, D8:243, 1983.

[32] J.O. Stiegler and K. Farrell. Scr. Metall., 8, 1974.

[33] Z. Suo and W. Lu. Forces that drive self-assembly on solid surfaces. J. Nanoparticles Res., 2000.

[34] H. Trinkaus, B.N. Singh, and A.J.E. Foreman. Mechanisms for decoration of dislocations by small dislocation loops under cascade damage conditions. J. Nucl. Mater., 249:91, 1997.

[35] H. Trinkaus, B.N. Singh, , and A.J.E. Foreman. J. Nucl. Mater., 251:172, 1997.

[36] Lauzeral J. Walgraef, D. and N.M. Ghoniem. Phys. Rev. B, 53:14782, 1996.

[37] J.E. Westmoreland, Sprague J.A., Smidt F.A., and Malmberg P.R. Rad. Eff., 26:1, 1975.

[38] J.B. Whitley. Ph.d thesis, Nuclear Engineering Department, University of Wisconsin, 1978.

[39] F.W. Wiffen. Proceedings of the International Conference on Radiation-Induced Voids in Metals, Albany, NY, June 1971, CONF-710601, Corbett, J.W. and Iannello, L.C. (Eds.), National Technical Information Service, Springfield, VA, page 386, 1972.

[40] S.J. Zinkle and L.L. Snead. J. Nucl. Mater., 225:132, 1995.

[41] 